Musées, Patrimoine et Culture scientifiques et techniques

$153 \mid 2014$

mai-juin 2014

\title{
Matériel d'étude et circulaire du 19 juillet 2012 dans les muséums
}

Nathalie Mémoire pour la Conférence Permanente des Muséums de France

\section{OpenEdition \\ Journals}

Édition électronique

URL : http://journals.openedition.org/ocim/1391

DOI : 10.4000/ocim.1391

ISSN : 2108-646X

Éditeur

OCIM

Édition imprimée

Date de publication : 25 juin 2014

ISSN : 0994-1908

Référence électronique

Nathalie Mémoire pour la Conférence Permanente des Muséums de France, « Matériel d'étude et circulaire du 19 juillet 2012 dans les muséums », La Lettre de l'OCIM [En ligne], 153 | 2014, mis en ligne le 25 juin 2016, consulté le 19 avril 2019. URL : http://journals.openedition.org/ocim/1391 ; DOI : 10.4000/ocim. 1391 


\title{
Matériel d'étude et circulaire du 19 juillet 2012 dans les muséums
}

\author{
Nathalie Mémoire \\ pour la Conférence Permanente des Muséums de France *
}

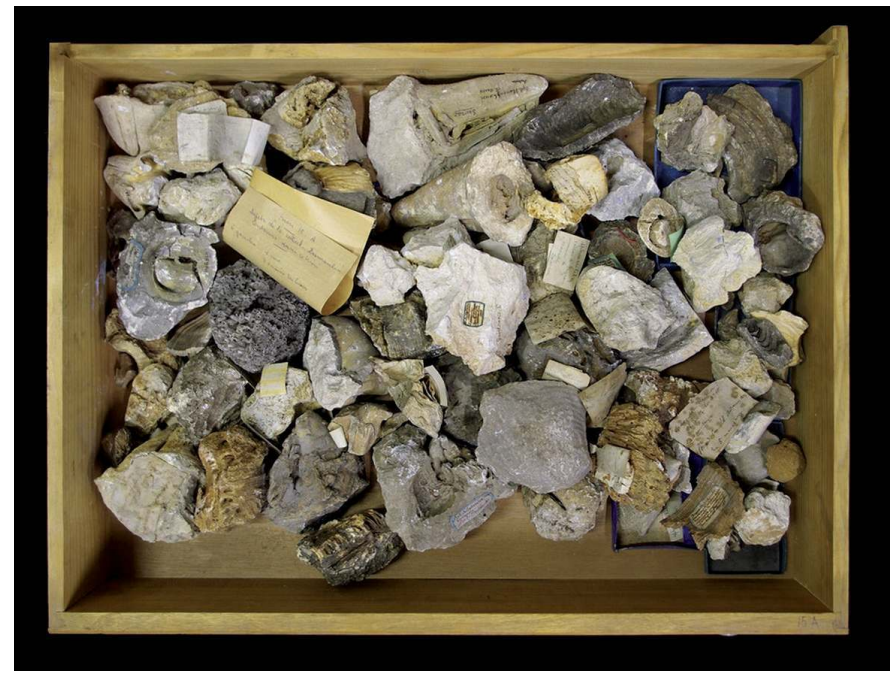

Comment considérer les accumulations passées telles que celles de ce tiroir de fossiles du muséum de Bordeaux ? Y sont mélangées des pièces visiblement documentées par leurs étiquettes encore collées et des pièces non documentées et non déterminées. Ensemble complexe à inventorier globalement ou matériel d'étude à trier? ๑) Muséum d'Histoire naturelle de Bordeaux/Laurent Charles

\footnotetext{
* Nathalie Mémoire est conservateur en chef du patrimoine, directrice du muséum d'Histoire naturelle de Bordeaux
} n.memoire@mairie-bordeaux.fr
Les résultats d'une enquête menée sur l'analyse patrimoniale du matériel d'étude dans les muséums d'Histoire naturelle permettent de préciser les différentes stratégies envisagées, de mesurer les difficultés rencontrées et de plaider en faveur d'un allongement des délais pour l'application de cette directive.

Les muséums d'Histoire naturelle sont diversement concernés par la circulaire du 19 juillet 2012 sur le matériel d'étude selon la nature et l'histoire de leurs collections.

Une journée de travail consacrée à l'interprétation de cette circulaire a été organisée le 18 octobre 2013 à destination des musées nationaux. Il ma été demandé d'y intervenir au titre de la CPMF (Conférence Permanente des Muséums de France) afin de présenter la lecture que nous en faisions dans les muséums. J'ai donc adressé, durant l'été 2013, un questionnaire destiné à donner un aperçu des interprétations et utilisations de ces nouvelles directives dans la dernière ligne droite du récolement. Vingt et un des trenteneuf musées représentés à la CPMF ont répondu, soit un peu plus de la moitié, ce qui, en cette période estivale peu favorable, montre le réel intérêt et l'implication des personnels des établissements.

\section{Importance du matériel d'étude}

par rapport à l'ensemble des fonds conservés

Ainsi, pour plus de la moitié d'entre eux, le matériel d'étude est numériquement estimé entre 5 et $15 \%$ du total des collections, moins de $1 \%$ pour l'un d'entre eux, mais entre 30 et $98 \%$ pour un tiers des musées, tandis que deux ne savent pas l'estimer. Ces importantes différences d'un établissement à l'autre sont certainement 
explicables par une différence de choix stratégique dans le traitement des accumulations passées, ainsi que nous le verrons par la suite.

\section{Origine du matériel d'étude des muséums}

Ce matériel est en effet très majoritairement issu d'accumulations passées (pour $90 \%$ d'entre eux). Elles représentent alors plus de $50 \%$ du total du matériel d'étude pour les trois quarts des musées tandis que près de $20 \%$ ne savent pas les estimer. Seuls deux musées ne sont pas concernés. On constate, mais en moindre proportion, que le produit de fouilles est cité pour la moitié des réponses.

Les collectes de dépouilles en attente de taxidermie ou de montages sont mentionnées par tous mais elles ne concernent qu'une quantité marginale de spécimens. À noter que presque tous les muséums tiennent pour celles-ci des registres papier ou des fichiers informatisés, mais un seul fait parapher par l'élu de sa collectivité en charge de la culture ainsi qu'il est préconisé dans la circulaire.

Échantillonnages, missions et voyages sont mentionnés à chaque fois par un tiers des musées mais pour moins de $1 \%$ du matériel.

\section{Choix stratégique pour les accumulations passées : matériel d'étude ou ensembles complexes?}

Il est probable que ces écarts de proportions du matériel d'étude par rapport au total des fonds ne sont pas uniquement dûs à la nature de ces fonds, mais également au sens donné au terme «matériel d'étude ». En effet, certains collègues y englobent des accumulations non encore inventoriées mais composées d'éléments documentés (par étiquettes associées) tandis que d'autres les ont plutôt considérées comme « ensembles complexes » de matériel patrimonial et récolés comme tels. Cette problématique a notamment été abordée lors de la journée de travail de mars 2013 à Paris qui a réuni treize muséums de la CPMF.

Dans le premier cas, une série ancienne considérée comme «matériel d'étude » ne figure pas au sein des collections «Musée de France » du musée. Elle n'est donc pas récolée mais décrite sommairement ; elle devra sous deux ans faire l'objet d'un plan prévisionnel d'étude et d'analyse patrimoniale permettant d'effectuer le tri en vue de son affectation.

Dans le second cas, la série est déjà considérée comme collection « Musée de France » et récolée comme un bien unique dit « ensemble complexe».

La réalisation de l'inventaire par lots simples ou par unités est mentionnée dans les suites à donner au récolement; il n'y a pas de tri ultérieur.
Néanmoins, le choix de la stratégie à adopter reste délicat pour les ensembles «mixtes », comprenant à la fois des unités documentées et des unités non identifiées ou non documentées, au sein d’une même série.

\section{L'analyse patrimoniale : résultats et intentions}

Huit musées déclarent avoir déjà effectué "l'analyse patrimoniale pour destination de ces collections ", et deux d'entre eux envisagent de la poursuivre après 2014. Sur les treize qui ne l'ont pas effectuée, seulement six envisagent de l'entreprendre après 2014 et encore, certains sont-ils très réservés, préférant se consacrer à la poursuite des inventaires rétrospectifs de leurs collections patrimoniales. Pour certains, la difficulté réside dans la parution tardive de la circulaire qui les a conduits, dans les années précédentes, à intégrer dans les collections « Musées de France » des ensembles qui auraient dû être traités dans les catégories proposées par la circulaire. Ceux qui ont effectué cette analyse précisent que, si l'analyse est relativement aisée à réaliser sur des ensembles issus de collectes raisonnées récentes, généralement documentées, elle est beaucoup plus délicate dans le cas des accumulations passées, lorsque ces données sont absentes ou très partielles.

À l'issue de cette analyse, six des huit musées qui l'ont réalisée ont versé entre 60 et $90 \%$ du matériel en collections « Musée de France » (après présentation en

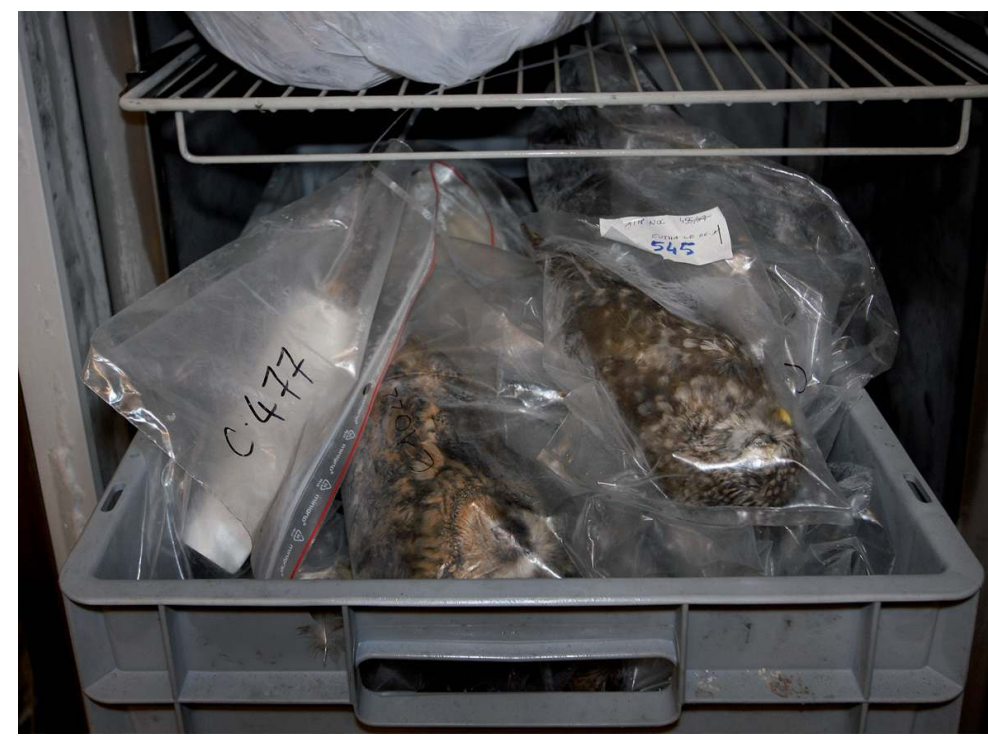

Les dépouilles en attente de montage (taxidermique ou ostéologique) représentent numériquement rarement plus de $1 \%$ du matériel d'étude des muséums. Elles sont généralement numérotées et référencées dans des registres particuliers. Au muséum de Bordeaux, le registre est informatisé et mis à jour au fur et à mesure des entrées et des sorties. ๑) Muséum d'Histoire naturelle de Bordeaux/Hana Goodall 
Commission scientifique régionale d'Acquisition) ; deux n'ont pas donné de proportion et deux n'ont pas présenté en CSRA, considérant que c'était sans objet pour des fonds anciens.

Pour les musées qui n'ont pas encore effectué ce tri mais l'envisagent, presque tous ont réfléchi à la destination de leur reliquat. Ont donc été regroupées les réponses de ceux qui ont réalisé le tri et les intentions de ceux qui l'envisagent, soit au total vingt musées ayant répondu. Il faut souligner qu’aucun établissement n'envisage d'aliéner du matériel à titre onéreux (possibilité mentionnée dans la circulaire). Toutes les autres possibilités sont réalisées ou envisagées, dans des proportions variables : en tête, l'élimination et l'utilisation du reliquat pour usage pédagogique, dans les deux cas pour $75 \%$ des réponses. Dans les autres affectations "pour usage », l'utilisation pour prélèvement pour analyse (ADN ou autre) est mentionnée par un tiers des musées tandis que l'utilisation pour restauration n’est envisagée que par deux établissements. La moitié des musées prévoit un reliquat "à préserver ", pour destination muséographique ou documentaire. Trois muséums seulement envisagent le transfert à un autre musée de France ou un établissement de recherche.

Les motifs de l'élimination sont les suivants : matériel non renseigné, en mauvais état, espèces communes ; peuvent s'y ajouter des critères d'encombrement et de risques d'infestation. Mais l'élimination n'est envisagée que très prudemment, si l'objet ne présente d'intérêt ni scientifique, ni historique, ni muséographique, ni pédagogique. À noter que, même en mauvais état, un matériel intéressant au plan scientifique ou historique peut être conservé. Les quantités déclarées éliminées représentent de 15 à $30 \%$ du matériel effectivement trié.
Plus de la moitié des établissements qui n'ont pas commencé le tri expriment le désir de participer à une journée d'étude pour les aider dans ces choix. Un délai de 5 ans pour mener les analyses patrimoniales semble insuffisant à la majorité des collègues, principalement en raison des volumes d'accumulations passées à résorber en regard des personnels affectés et des autres tâches prioritaires à mener sur les collections « Musée de France », mais sont aussi évoquées les difficultés à documenter ces accumulations ou à trouver les compétences dans chaque spécialité.

En conclusion, en ce qui concerne la circulaire ellemême, les avis sont partagés puisqu'une moitié des collègues pense qu'elle répond complètement ou en partie aux problématiques des collections des muséums en donnant un cadre, tandis que l'autre moitié estime que la terminologie ne paraît pas toujours suffisamment claire et que l'application aux accumulations passées reste difficile. 